Article

\title{
Synthesis of Antibacterial Gelatin/Sodium Alginate Sponges and Their Antibacterial Activity
}

\author{
Yanyi Wen ${ }^{1,2}$, Bing $\mathrm{Yu}^{2}$, Zhongjie Zhu ${ }^{2}$, Zhuoran Yang ${ }^{2}$ and Wei Shao ${ }^{1,2, *}$ \\ 1 Jiangsu Co-Innovation Center of Efficient Processing and Utilization of Forest Resources, \\ Nanjing Forestry University, Nanjing 210037, China; wenyanyis@163.com \\ 2 College of Chemical Engineering, Nanjing Forestry University, Nanjing 210037, China; \\ yubing1007@163.com (B.Y.); 13382367651@163.com (Z.Z.); y1693383415@163.com (Z.Y.) \\ * Correspondence: W.shao@njfu.edu.cn
}

Received: 31 July 2020; Accepted: 20 August 2020; Published: 26 August 2020

check for updates

\begin{abstract}
In the present study, sponges with the antibiotic tetracycline hydrochloride (TCH) loaded into alginate incorporated with gelatin (G/SA) were fabricated. The G/SA sponges were characterized by scanning electron microscopy (SEM), Fourier transform infrared spectroscopy (FTIR) and thermogravimetric (TG) analysis. G/SA sponges show a three-dimensional network structure with high porosity. An excellent swelling behavior and a controlled $\mathrm{TCH}$ release performance are observed from G/SA sponges. Moreover, they exhibit good antibacterial activity against both Gram-positive and Gram-negative bacteria.
\end{abstract}

Keywords: alginate; gelatin; porosity; antibacterial

\section{Introduction}

As the largest organ, the skin acts as a barrier against the invasion of the human body by pathogens [1]. Once it is injured, however, it becomes particularly susceptible to bacterial infections. It was reported that mortality in burn patients associated with bacterial infections is up to 75\% [2]. Therefore, wound dressing materials with infection prevention, elimination of excess exudates, air permeation and hemostasis are in high demand for the treatment of damaged skin [3-5].

Natural biopolymers including chitosan, alginate, cellulose, collagen, hyaluronic acid and gelatin in the form of films, sponges and fibers have been applied in wound dressing applications [6-8]. Among these biopolymers, sodium alginate (SA) extracted from brown seaweeds is one of the most commonly used biomaterials owing to its nontoxicity, facile production, biodegradability, low cost and biocompatibility. Therefore, SA has many applications such as biomedical, food packaging, pharmaceutical and cosmetic fields $[9,10]$. However, SA has some limitations in the process of non-spherical forms due to its rigid and fragile nature. Blending SA with another biopolymer is considered to be an ideal solution to overcome this drawback. SA blended with chitosan loaded with gentamicin was fabricated via the electrospinning method to be used as a wound dressing nanofibrous material with good antibacterial property and enhanced skin regeneration [6]. Chitin/chitosan and fucoidan were added to SA to develop a functional wound dressing for the repair of healing-impaired wounds [11]. A SA/aloe vera/cellulose nanocrystals composite film was prepared by a solvent casting technique and it was demonstrated to effectively inhibit the growth of Staphylococcus aureus bacterial colonies, thus showing potential for wound dressing applications [12].

Gelatin is a water-soluble denatured protein derived from skin, tendons or bones [13]. It possesses many advantages such as abundance, low cost, renewability, excellent film-forming ability, good absorption capacity and great biocompatibility [14]. Therefore, it finds wide application in various fields such as the food industry, water treatment, pharmaceuticals, drug delivery, scaffolds and 
wound dressings [15]. Gelatin-based composites exhibit great potential in wound dressings and tissue engineering since they can provide an essential microenvironment for tissue growth and excellent water absorbance capacity [16]. Gelatin/dextran-maleic anhydride composite fibers were prepared via electrospinning and photocrosslinking methods and the resulting composite fibers could support cell proliferation and adhesion, showing potential in tissue engineering [17]. A bilayered human amniotic epithelial cells and Wharton's jelly-derived mesenchymal stem cells-laden alginate/gelatin composite hydrogel was fabricated by a 3D bioprinting method. This represents a potential preparation method for skin substitutes [18]. Konjac/gelatin hydrogel was fabricated by alkali processing and thermal treatment and it showed excellent matrine encapsulation efficiency. Moreover, matrine-loaded konjac/gelatin hydrogel could not only maintain a physiological environment beneficial to wound healing, but also inhibit the growth of bacteria on the wound surface [19]. A novel multifunctional poly( $\gamma$-glutamic acid)/gelatin hydrogel was fabricated and confirmed to be a promising wound dressing material for the wound healing [20]. Dialdehyde carboxymethylcellulose crosslinked gelatin/polyethylene glycol composite hydrogel fiber was prepared and shown to be well-suited for wound dressing applications with low cytotoxicity [21].

In this work, gelatin was incorporated into SA to enhance the shape forming properties and tetracycline hydrochloride (TCH) was then loaded to fabricate antibacterial gelatin/SA (G/SA) composite sponges. TCH is one of the most widely applied cationic antibiotics and it is effective for various bacterial infection treatments, such as periodontal, skin and bone, urinary and acnes [22,23]. The morphologies of the prepared G/SA sponges were investigated by scanning electron microscopy (SEM) and they were further characterized by Fourier transform infrared (FTIR), Thermogravimetric (TG) analysis, porosity and swelling behavior determinations. The TCH release profile was revealed by an in vitro release study in phosphate buffer saline (PBS) buffer solution at pH 7.4. Antimicrobial activity against Gram-negative E. coli ATCC 25,922 and B. subtilis ATCC 9372, Gram-positive S. aureus ATCC 6538 and P. aeruginosa ATCC 27,853 was determined by measuring the zone of inhibition.

\section{Materials and Methods}

\subsection{Materials}

Gelatin from porcine skin (type A, gel strength $300 \mathrm{~g}$ Bloom) was purchased from Sigma-Aldrich (St. Louis, MO, USA). Tetracycline hydrochloride (TCH, $>98 \%$ ) was purchased from EKEAR Bio@Tech Co. Ltd. (Shanghai, China). SA was purchased from Aladdin Chemical Co. Ltd. (Shanghai, China). The other chemicals used in the tests were purchased from Sinopharm Chemical Reagent Co., Ltd. (Shanghai, China). All reagents were analytical grade and used as received without further purification.

\subsection{Synthesis of $G / S A$ Composite Sponges}

Firstly, a $2 \%$ SA solution, $1 \%$ gelatin solution and $2 \% \mathrm{CaCl}_{2}$ solution were prepared, respectively. SA solution and gelatin solution were mixed with a weight ratio of 1:1 to prepare G/SA mixture. Then $10 \mathrm{~g} \mathrm{G} / \mathrm{SA}$ mixture was poured onto a glass plate with the diameter of $60 \mathrm{~mm}$, and cross-linked by $\mathrm{CaCl}_{2}$ solution for $24 \mathrm{~h}$. TCH was dissolved in de-ionized (DI) water to achieve different concentrations of $0.005,0.01,0.05,0.1$ and $0.2 \mathrm{mg} / \mathrm{mL}$, respectively. The obtained G/SA hydrogels were placed to $40 \mathrm{~mL}$ TCH solutions for $24 \mathrm{~h}$ in the dark. Then the TCH-loaded G/SA hydrogels were taken out and rinsed with DI water to remove any unloaded TCH and the excessive $\mathrm{CaCl}_{2}$, and freeze-dried at $-40{ }^{\circ} \mathrm{C}$ for $24 \mathrm{~h}$. The obtained TCH loaded sponges obtained from immersing them in different concentrations of TCH solutions are marked as G/SA1, G/SA2, G/SA3, G/SA4 and G/SA5.

\subsection{Characterization}

A JSM-7600F SEM (JEOL, Tokyo, Japan) was used to investigate the surface morphologies of G/SA and G/SA5 sponges. FTIR spectra were recorded on a Spectrum Two Spectrometer (Perkin Elmer, 
Akron, OH, USA). TG analysis was carried out using a model Q5000 TGA (TA Instruments, New Castle, DE, USA) with a heating rate of $10^{\circ} \mathrm{C} / \mathrm{min}$ under nitrogen atmosphere.

\subsection{Swelling Assays and Porosity Determination}

The swelling behaviors of the sponges were determined through a gravimetric method. First, the sponges were cut into round pieces with the diameter of $10 \mathrm{~mm}$ and their dry weights $\left(\mathrm{W}_{0}\right)$ were accurately measured. Each sample was immersed in DI water at room temperature. The swollen sponges were withdrawn from the water and their wet weights of the swollen sponges $\left(\mathrm{W}_{1}\right)$ was measured after the removal of excess surface water by gently blotting with a filter paper. The swelling ratio of the sponges was calculated as follows:

$$
\text { Swelling ratio }=\frac{\mathrm{W}_{1}-\mathrm{W}_{0}}{\mathrm{~W}_{0}} \times 100 \%
$$

The porosity was determined using an immersing method in the ethanol until saturation. The samples with the diameter of $10 \mathrm{~mm}$ were weighed before and after immersion into the ethanol. All testing was proceeded in triplicate. The porosity was calculated according to Equation (2):

$$
\text { Porosity }=\frac{W_{2}-W_{1}}{\rho V} \times 100 \%
$$

where $W_{1}$ and $W_{2}$ are the weights of tested sponges before and after immersion in the ethanol, $\rho$ is the density of ethanol and $V$ is the volume of the sponge.

\subsection{TCH Loading Calculation}

$\mathrm{TCH}$ loadings were calculated according to the original concentration of $\mathrm{TCH}\left(\mathrm{C}_{0}\right)$ and the concentration of unloaded TCH $\left(\mathrm{C}_{1}\right)$ determined by a Spectrum Two Spectrometer (Perkin Elmer, Akron, OH, USA) at the monitoring wavelength of $356 \mathrm{~nm}$. TCH loadings (W) in the G/SA sponges were calculated using the following equation:

$$
\mathrm{W}=\frac{\mathrm{C}_{0}-\mathrm{C}_{1}}{\mathrm{~A}} \times \mathrm{V}
$$

where $\mathrm{V}$ is the volume of total TCH solution, $\mathrm{A}$ is the area of the G/SA sponge.

\subsection{TCH Release Behaviors}

The TCH release behaviors were studied in $0.1 \mathrm{M}$ phosphate buffer saline (PBS) buffers with $\mathrm{pH}$ 7.4. The sponges were cut into round pieces with the diameter of $10 \mathrm{~mm}$. The tested samples were fully immersed in a beaker containing $50 \mathrm{~mL}$ PBS at $37^{\circ} \mathrm{C}$ and placed in a dark place. At specific time points, an aliquot of $3.5 \mathrm{~mL}$ was collected from each solution and the absorbance was then measured at $356 \mathrm{~nm}$ on a Spectrum Two Spectrometer (Perkin Elmer, Akron, OH, USA). An equivalent volume of fresh PBS buffer was replaced into the system after each sampling to maintain constant medium volume. Thus, the concentrations of released TCH obtained at different times can be calculated. As a result, the cumulative released TCH amounts can be calculated accordingly. The experiments were performed in triplicate.

\subsection{Antibacterial Activity}

The antibacterial activities of G/SA sponges were investigated against Gram-negative E. coli ATCC 25,922 and B. subtilis ATCC 9372, Gram-positive S. aureus ATCC 6538 and P. aeruginosa ATCC 27,853 by disk diffusion method. G/SA sponges were cut into round shapes with $10 \mathrm{~mm}$ diameter and sterilized by ultraviolet lamp for $60 \mathrm{~min}$. Lawns of test bacteria (about $1 \times 10^{5} \mathrm{CFU} / \mathrm{plate}$ ) were prepared on TSA. The sterilized samples were then carefully placed upon the lawns. The plates were placed in a 
$37^{\circ} \mathrm{C}$ incubator for $24 \mathrm{~h}$. Then inhibitory action was determined by measuring the diameter of the inhibition zones.

\subsection{Statistical Analysis}

The obtained data are expressed as mean \pm standard error (SE). Statistical differences were evaluated using a Student's t-test. A $p$-value of $<0.05$ was considered to be statistically significant.

\section{Results and Discussion}

\subsection{Surface Morphology}

The exudate absorption capacity is directly affected by the surface morphology and porosity of polymer-based wound dressings, so the morphologies of G/SA and TCH-loaded G/SA (G/SA5) sponges were studied using SEM (Figure 1). Figure 1A,B show the cross-sectional morphologies of G/SA sponge with different magnifications. It can be clearly seen that G/SA sponge exhibits a highly porous three-dimensional network structure with interconnected pores throughout the hybrid sponge. After TCH was loaded into the sponge, small difference in pores shape and size can be found although G/SA5 sponge still keeps its 3D structure, as shown in Figure 1C,D.

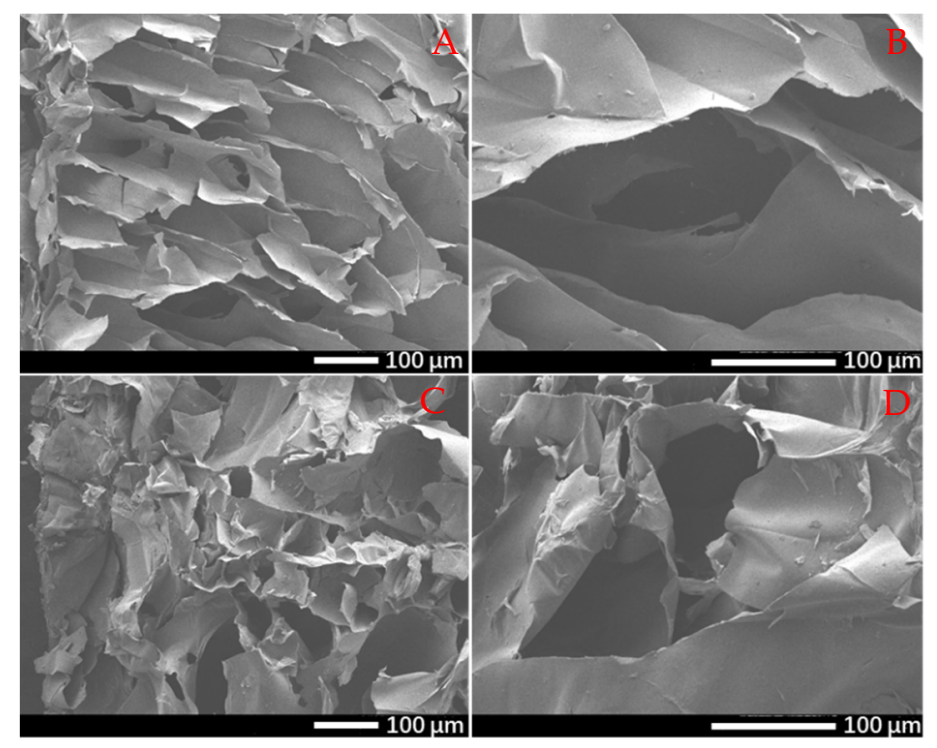

Figure 1. SEM images of G/SA (A,B) and G/SA5 (C,D) sponges.

Figure 2 shows the porosities of G/SA and TCH loaded G/SA sponges. The G/SA sponge shows a relatively high porosity of $90.2 \%$. After TCH was loaded into G/SA sponges, a slight decrease in porosity to a range of $87.1-88.5 \%$ can be observed. TCH loadings of G/SA composite sponges are listed in Table 1. It can be clearly found that TCH loading increases with the increase of initial TCH concentration. These high porosities benefit for exudates absorbing, making the fabricated sponges show great potentials in wound dressings application.

Table 1. TCH loadings of G/SA composite sponges.

\begin{tabular}{ccc}
\hline & $\begin{array}{c}\text { Initial TCH } \\
\text { Concentration }(\mathbf{m g} / \mathbf{m L})\end{array}$ & $\begin{array}{c}\text { TCH Loading } \\
\left(\mathbf{m g} / \mathbf{c m}^{2}\right)\end{array}$ \\
\hline G/SA1 & 0.005 & 0.081 \\
G/SA2 & 0.01 & 0.100 \\
G/SA3 & 0.05 & 0.516 \\
G/SA4 & 0.1 & 0.873 \\
G/SA5 & 0.2 & 1.463 \\
\hline
\end{tabular}




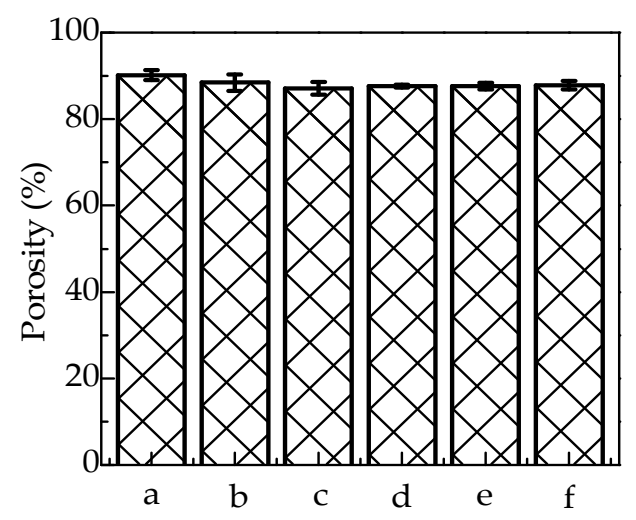

Figure 2. Porosities of G/SA and TCH loaded G/SA sponges (a:G/SA, b:G/SA1, c:G/SA2, d:G/SA3, e:G/SA4 and f:G/SA5).

\subsection{Thermal Properties}

The TG and derivative thermogravimetric (DTG) curves of SA, gelatin and G/SA5 are shown in Figure 3. Three weight loss stages were found in the TG curve of SA (curve a in Figure 3A). The first weight loss stage occurs below $120^{\circ} \mathrm{C}$, which is attributed to the evaporation of absorbed water. The second weight loss occurred in the range of $200-350{ }^{\circ} \mathrm{C}$ involving the degradation of main backbone of SA and the formation of sodium carbonate. The third weight loss stage starting from $350{ }^{\circ} \mathrm{C}$ to $730^{\circ} \mathrm{C}$ is the decomposition of both SA and sodium carbonate [24,25]. The residue of SA is $30.8 \%$, corresponding to the formed carbon dioxide and calcium oxide [26]. Thermal degradation of gelatin occurs in two stages (curve $b$ in Figure 3A). The first stage occurs from room temperature up to $180{ }^{\circ} \mathrm{C}$ and the second stage occurs between $180{ }^{\circ} \mathrm{C}$ and $370^{\circ} \mathrm{C}$ corresponding to the degradation of protein [27].
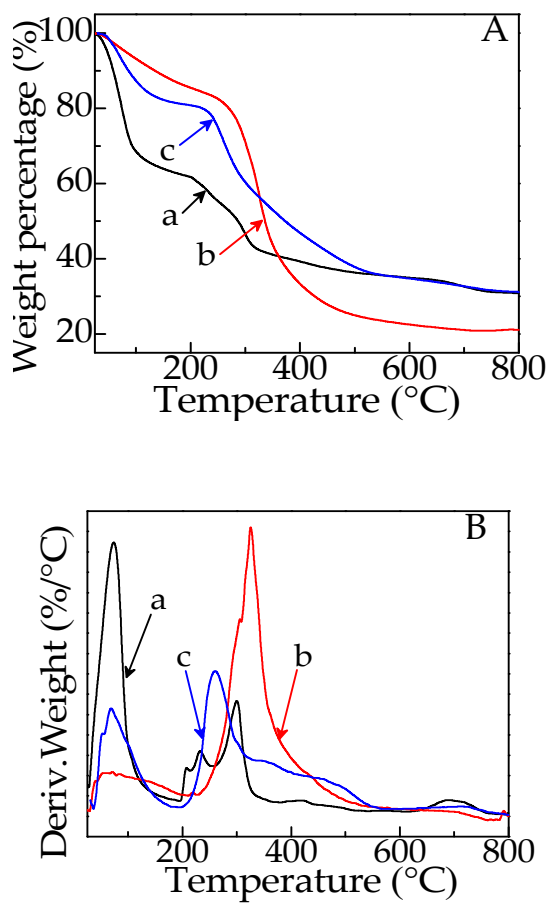

Figure 3. TG (A) and DTG (B) curves of SA (a), gelatin (b) and G/SA5 (c).

In the case of G/SA5 sponge (curve c in Figure $3 A$ ), two weight loss stages were shown. The first one is the loss of adsorbed water which occurs below $200^{\circ} \mathrm{C}$. The second one is the complex decomposition 
of SA, gelatin, sodium carbonate and TCH in the range of $200-780{ }^{\circ} \mathrm{C}$. The residue of G/SA5 is $31.2 \%$, which is higher than those of SA and gelatin $(21.1 \%)$ due to the existence of TCH. Therefore, TCH are demonstrated successfully to be incorporated into G/SA sponges.

\subsection{FTIR Analysis}

FTIR spectra of G/SA, G/SA1, G/SA2, G/SA3, G/SA4, G/SA5 and TCH are given in Figure 4. In the case of G/SA (curve a), characteristic bands located at $3328 \mathrm{~cm}^{-1}, 1559 \mathrm{~cm}^{-1}$ correspond to the stretching of $-\mathrm{NH}$ and $-\mathrm{OH}$ groups, and the $\mathrm{N}-\mathrm{H}$ deformation (amide II) of gelatin [28]. Moreover, the peak at $1624 \mathrm{~cm}^{-1}$ is assigned to the $\mathrm{C}=\mathrm{O}$ stretching (amide I) of gelatin and the asymmetric $\mathrm{COO}$ stretching vibration of SA. The absorption band at $1419 \mathrm{~cm}^{-1}$ corresponds to the symmetric COO stretching vibration of SA $[13,29]$. The asymmetric and symmetric COO stretching vibrations of the reported SA without any cross-linking are located at 1612 and $1417 \mathrm{~cm}^{-1}$, respectively [30]. They show a blue shift due to the formation of ionic bonding between $-\mathrm{COO}^{-}$and $\mathrm{Ca}^{2+}$ in this study. In the FTIR spectrum of TCH (curve g), the peaks located at $1665 \mathrm{~cm}^{-1}, 1617 \mathrm{~cm}^{-1}, 1590 \mathrm{~cm}^{-1}, 1545 \mathrm{~cm}^{-1}$ and $1440 \mathrm{~cm}^{-1}$ are assigned to the $\mathrm{C}=\mathrm{O}$ vibration of amide $\mathrm{I}$, the $\mathrm{C}=\mathrm{O}$ vibration of $\mathrm{A}$-ring, the $\mathrm{C}=\mathrm{O}$ vibration of $\mathrm{C}$-ring, the $\mathrm{N}-\mathrm{H}$ deformation of amide II and the $\mathrm{C}=\mathrm{C}$ vibration of aromatic ring, respectively [31]. For TCH-loaded G/SA sponges (curves b-f), the intensity of peak at $1545 \mathrm{~cm}^{-1}$ increases with TCH loading raising although most characteristic peaks of TCH overlaps with the peaks of G/SA. Thus, the existence of TCH in the G/SA sponges is verified.

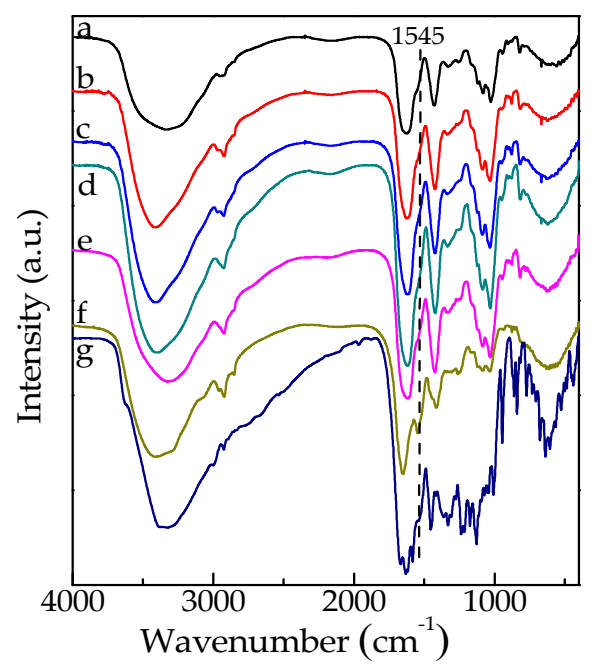

Figure 4. FTIR spectra of G/SA (a), G/SA1 (b), G/SA2 (c), G/SA3 (d), G/SA4 (e), G/SA5 (f) sponges and $\mathrm{TCH}(\mathrm{g})$.

\subsection{Swelling Behaviors In Vitro}

The water absorbing and holding abilities are essential characteristics for polymer-based wound dressings which facilitate the adsorption of exudates, body fluids or metabolites. Figure 5 shows the dynamic swelling performance of G/SA composite sponges. The swelling ratio of G/SA sponge is $2190 \%$ after $24 \mathrm{~h}$, which is the highest swelling ratio among the tested sponges. SA has many hydrophilic groups including carboxylic and hydroxyl groups, and gelatin also contains carboxylic and hydroxyl groups as well as amino groups, leading to a high swelling performance. Meanwhile, TCH loaded G/SA sponges show slightly reduced swelling performance with the swelling ratios ranging from 1780 to $1960 \%$ after $24 \mathrm{~h}$. This phenomenon could be due to the fact that the porosities of sponges were slightly decreased after being loaded with TCH. 


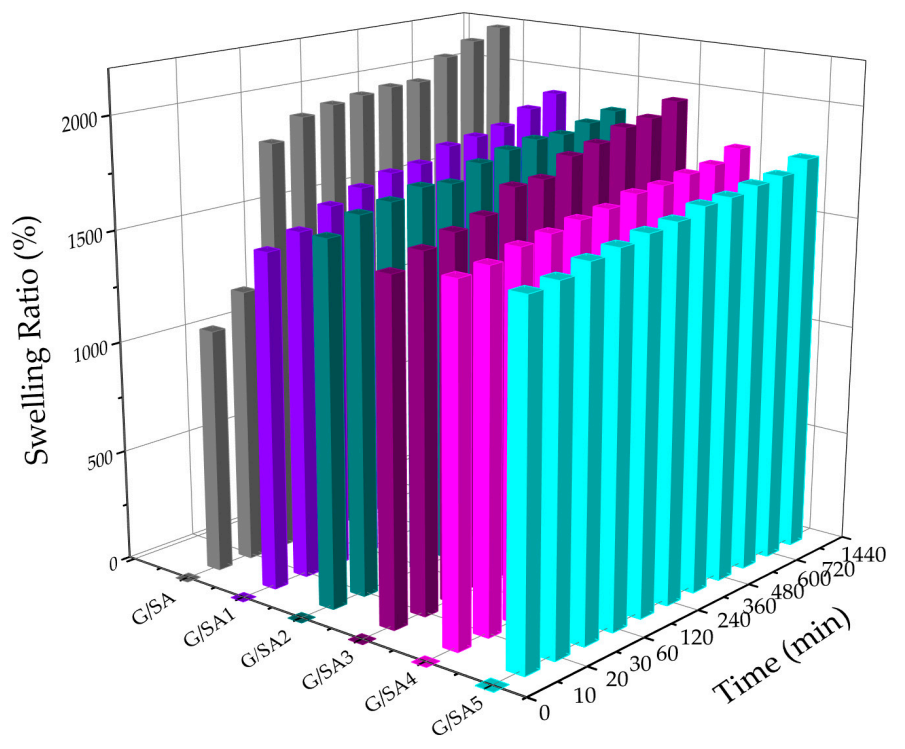

Figure 5. Swelling behaviors of G/SA composite sponges.

\subsection{TCH Release Behavior}

The TCH release behaviors were determined in $0.1 \mathrm{M}$ PBS buffer at $\mathrm{pH} 7.4$ and the result is shown in Figure 6A. A burst release behavior was displayed in the first $1 \mathrm{~h}$ for the TCH-loaded G/SA sponges and this behavior could be probably due to the accumulation of TCH on the surface of G/SA sponges. Then a gradual increased release behavior for the G/SA composite sponges was shown and the release behavior lasts for $12 \mathrm{~h}$. Moreover, the released amount is positively related to the TCH loadings in the G/SA sponges.
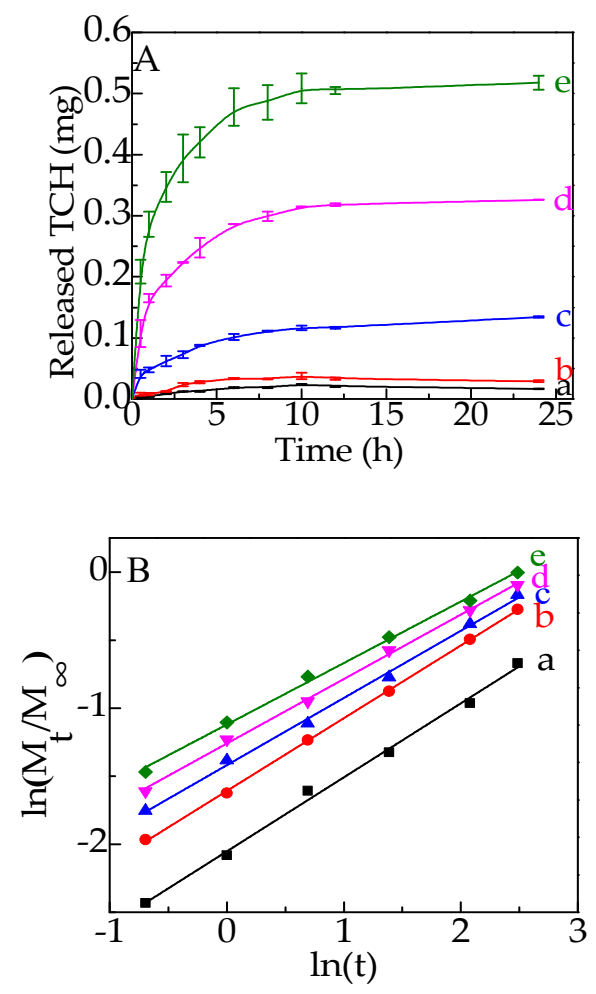

Figure 6. TCH release behavior of G/SA sponges (A) and plots of $\ln (\mathrm{Mt} / \mathrm{M})$ versus $\ln (\mathrm{t})$ (B). (a:G/SA1, b:G/SA2, c:G/SA3, d:G/SA4, e:G/SA5). 
Thus, G/SA composite sponge is considered to be a good TCH carrier with controlled release performance, demonstrating its potential wound dressing application in the treatment of infections and inflammation.

Four mathematical models, including zero order, first order, Higuchi and Korsmeyer-Peppas models were used to fit the TCH release profiles. The obtained results are shown in Table 2. The best fitting mathematical model for the TCH release mechanism was determined by the calculated correlation coefficient $\left(R^{2}\right)$. It can be found that the Korsmeyer-Peppas model is the best fitting one, which is a semi-experimental exponential function equation considering diffusion of drug and erosion of polymer matrix [16]. The plots of $\ln (\mathrm{Mt} / \mathrm{M})$ versus $\ln (\mathrm{t})$ using Korsmeyer-Peppas model are displayed in Figure $6 \mathrm{~B}$. The diffusional constant $\mathrm{n}$ indicates the drug release mechanism. In this study, $\mathrm{n}$ values lie between 0.45 and 0.89 , suggesting the TCH release behavior follows non-Fickian diffusion, which corresponds to the combined effects of the drug releases by dissolution, diffusion and erosion of matrix.

Table 2. $\mathrm{K}, \mathrm{n}$ and $\mathrm{R}^{2}$ values obtained from the mathematical modeling.

\begin{tabular}{|c|c|c|c|c|}
\hline \multirow{2}{*}{ Type of Model } & \multirow{2}{*}{ Name of Samples } & \multicolumn{3}{|c|}{ Fitting Parameters } \\
\hline & & $\mathbf{K}$ & $\mathbf{n}$ & $\mathbf{R}^{2}$ \\
\hline \multirow{5}{*}{$\begin{array}{l}\text { Korsmeyer-Peppas } \\
\qquad \mathrm{M}_{\mathrm{t}} / \mathrm{M}_{\infty}=\mathrm{Kt}^{\mathrm{n}}\end{array}$} & G/SA1 & 0.12836 & 0.54398 & 0.99805 \\
\hline & G/SA2 & 0.200118 & 0.53455 & 0.99986 \\
\hline & G/SA3 & 0.241837 & 0.49382 & 0.9987 \\
\hline & G/SA4 & 0.283569 & 0.47373 & 0.99919 \\
\hline & G/SA5 & 0.325983 & 0.452 & 0.99866 \\
\hline \multirow{5}{*}{$\begin{array}{c}\text { Zero-order } \\
\mathrm{M}_{\mathrm{t}} / \mathrm{M}_{\infty}=\mathrm{Kt}\end{array}$} & G/SA1 & 0.06694 & / & 0.91416 \\
\hline & G/SA2 & 0.06119 & / & 0.69879 \\
\hline & G/SA3 & 0.07117 & / & 0.97968 \\
\hline & G/SA4 & 0.04232 & / & 0.94553 \\
\hline & G/SA5 & 0.04489 & / & 0.87162 \\
\hline \multirow{5}{*}{$\begin{array}{c}\text { First-order } \\
\mathrm{M}_{\mathrm{t}} / \mathrm{M}_{\infty}=1-\mathrm{e}^{-\mathrm{Kt}}\end{array}$} & G/SA1 & 0.24059 & / & 0.98953 \\
\hline & G/SA2 & 0.31313 & / & 0.92621 \\
\hline & G/SA3 & 0.41652 & / & 0.90066 \\
\hline & G/SA4 & 0.7134 & 1 & 0.88187 \\
\hline & G/SA5 & 0.91565 & I & 0.8789 \\
\hline \multirow{5}{*}{$\begin{array}{c}\text { Higuchi } \\
\mathrm{M}_{\mathrm{t}} / \mathrm{M}_{\infty}=\mathrm{Kt}^{1 / 2}\end{array}$} & G/SA1 & 0.2986 & / & 0.9315 \\
\hline & G/SA2 & 0.36825 & / & 0.87818 \\
\hline & G/SA3 & 0.2243 & / & 0.96647 \\
\hline & G/SA4 & 0.22741 & 1 & 0.94 \\
\hline & G/SA5 & 0.20267 & / & 0.90434 \\
\hline
\end{tabular}

\subsection{Antibacterial Activity}

The antibacterial activities of G/SA composite sponges towards Gram-negative E. coli ATCC 25,922 and B. subtilis ATCC 9372, Gram-positive S. aureus ATCC 6538 and P. aeruginosa ATCC 27,853 were investigated by the disc diffusion method. The antibacterial activity is determined by measuring the clear zone of inhibition around the samples after $24 \mathrm{~h}$ incubation (Figure 7). For G/SA without any $\mathrm{TCH}$ loading, no inhibition zones were observed around the tested four strains, implying that G/SA sponge does not have any antibacterial activities. While, G/SA composite sponges have different levels of antibacterial activities. The measured average diameters of inhibition zones are shown in Figure 8. It can be clearly seen that the inhibition zone increases with the increase of TCH loading in the G/SA sponge. Moreover, G/SA composite sponges show a good antibacterial activity against E. coli, S. aureus and $B$. subtilis. However, little antibacterial activity against $P$. aeruginosa was shown. The differences in the antibacterial activity could be due to the susceptibility difference against different strains of the loaded TCH. Therefore, G/SA composite sponges show good antibacterial activities, which makes them have great potentials in the antibacterial wound dressing materials applications. 


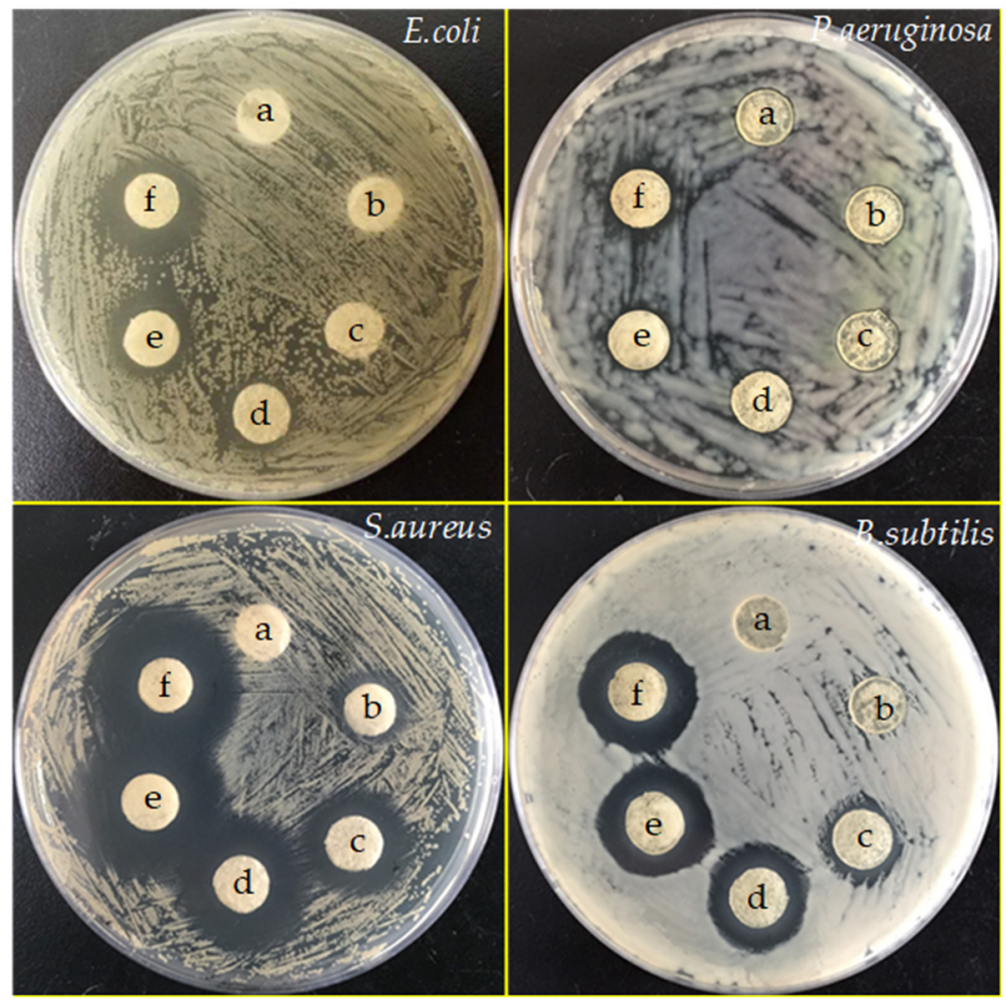

Figure 7. Inhibition zone pictures of G/SA composite sponges: G/SA (a), G/SA1 (b), G/SA2 (c), G/SA3 (d), G/SA4 (e), G/SA5 (f) sponges.

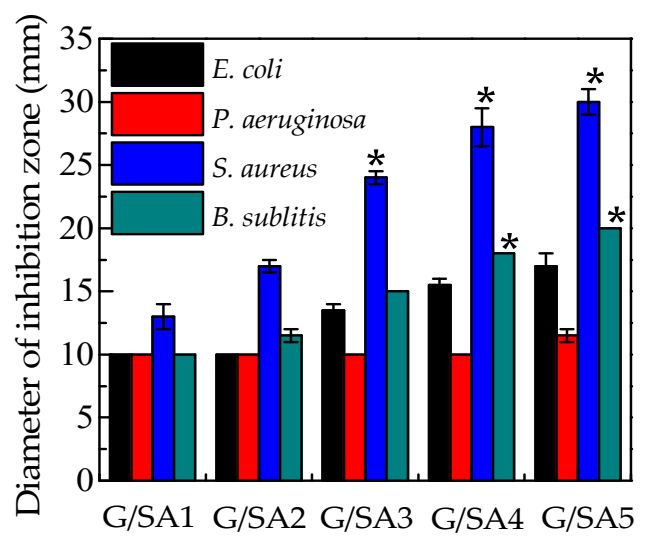

Figure 8. Diameters of inhibition zone of G/SA composite sponges against different strains. $\left({ }^{*} p<0.05\right)$.

\section{Conclusions}

In summary, TCH-loaded G/SA sponges were successfully synthesized and they display good swelling behavior and high porosity. G/SA composite sponges exhibit three-dimensional network structures, which help the G/SA sponges to facilitate TCH loading. The TCH-loaded G/SA sponges show controlled release performance and good antibacterial effects against E. coli ATCC 25,922 and B. subtilis ATCC 9372 and S. aureus ATCC 6538. Thus, the fabricated G/SA sponges show potential as promising substrates for drug delivery applications in wound dressing and other biomedical applications.

Author Contributions: Conceptualization, W.S.; methodology, B.Y. and Z.Z.; investigation, Z.Y.; writing一original draft preparation, Y.W.; writing - review and editing, W.S. All authors have read and agreed to the published version of the manuscript. 
Funding: This work was funded by the Six Talents Peak Project of Jiangsu Province, the National College Students' Practice and Innovation Training Project (201910298013Z, 2020NFUSPITP0077) and the Priority Academic Program Development of Jiangsu Higher Education Institutions (PAPD).

Acknowledgments: The authors thank for Advanced Analysis and Testing Center of Nanjing Forestry University.

Conflicts of Interest: The authors declare no conflict of interest.

\section{References}

1. Varaprasad, K.; Jayaramudu, T.; Kanikireddy, V.; Toro, C.; Sadiku, E.R. Alginate-based composite materials for wound dressing application:A mini review. Carbohydr. Polym. 2020, 236, 116025. [CrossRef] [PubMed]

2. Huebinger, R.M.; Stones, D.H.; de Souza, S.M.; Carlson, D.L.; Song, J.; Vaz, D.P.; Keen, E.; Wolf, S.E.; Orth, K.; Krachler, A.M. Targeting bacterial adherence inhibits multidrug-resistant Pseudomonas aeruginosa infection following burn injury. Sci. Rep. 2016, 6, 39341. [CrossRef]

3. Wang, S.; Yan, F.; Ren, P.; Li, Y.; Wu, Q.; Fang, X.; Chen, F.; Wang, C. Incorporation of metal-organic frameworks into electrospun chitosan/poly (vinyl alcohol) nanofibrous membrane with enhanced antibacterial activity for wound dressing application. Int. J. Biol. Macromol. 2020, 158, 9-17. [CrossRef]

4. Jamaledin, R.; Yiu, C.K.Y.; Zare, E.N.; Niu, L.N.; Vecchione, R.; Chen, G.; Gu, Z.; Tay, F.R.; Makvandi, P. Advances in Antimicrobial Microneedle Patches for Combating Infections. Adv. Mater. 2020, 32, e2002129. [CrossRef] [PubMed]

5. Wang, C.Y.; Makvandi, P.; Zare, E.N.; Tay, F.R.; Niu, L.N. Advances in Antimicrobial Organic and Inorganic Nanocompounds in Biomedicine. Adv. Ther. 2020, 3, 2000024. [CrossRef]

6. Bakhsheshi-Rad, H.R.; Hadisi, Z.; Ismail, A.F.; Aziz, M.; Akbari, M.; Berto, F.; Chen, X.B. In vitro and in vivo evaluation of chitosan-alginate/gentamicin wound dressing nanofibrous with high antibacterial performance. Polym. Test. 2020, 82, 106298. [CrossRef]

7. Wang, F.; Li, J.R.; Tang, X.Y.; Huang, K.X.; Chen, L. Polyelectrolyte three layer nanoparticles of chitosan/dextran sulfate/chitosan for dual drug delivery. Colloid Surf. B Biointerfaces 2020, 190, 7. [CrossRef]

8. Makvandi, P.; Ali, G.W.; Della Sala, F.; Abdel-Fattah, W.I.; Borzacchiello, A. Biosynthesis and characterization of antibacterial thermosensitive hydrogels based on corn silk extract, hyaluronic acid and nanosilver for potential wound healing. Carbohydr. Polym. 2019, 223, 115023. [CrossRef]

9. Gholizadeh, B.S.; Buazar, F.; Hosseini, S.M.; Mousavi, S.M. Enhanced antibacterial activity, mechanical and physical properties of alginate/hydroxyapatite bionanocomposite film. Int. J. Biol. Macromol. 2018, 116, 786-792. [CrossRef]

10. Wu, W.; Liu, T.; He, H.; Wu, X.; Cao, X.; Jin, J.; Sun, Q.; Roy, V.A.L.; Li, R.K.Y. Rhelogical and antibacterial performance of sodium alginate/zinc oxide composite coating for cellulosic paper. Colloids Surf. B Biointerfaces 2018, 167, 538-543. [CrossRef]

11. Murakami, K.; Aoki, H.; Nakamura, S.; Nakamura, S.-I.; Takikawa, M.; Hanzawa, M.; Kishimoto, S.; Hattori, H.; Tanaka, Y.; Kiyosawa, T.; et al. Hydrogel blends of chitin/chitosan, fucoidan and alginate as healing-impaired wound dressings. Biomaterials 2010, 31, 83-90. [CrossRef] [PubMed]

12. Thomas, D.; Nath, M.S.; Mathew, N.; Reshmi, R.; Philip, E.; Latha, M.S. Alginate film modified with aloevera gel and cellulose nanocrystals for wound dressing application: Preparation, characterization and in vitro evaluation. J. Drug Deliv. Sci. Technol. 2020, 59, 101894. [CrossRef]

13. Roy, S.; Rhim, J.-W. Preparation of antimicrobial and antioxidant gelatin/curcumin composite films for active food packaging application. Colloids Surf. B Biointerfaces 2020, 188, 110761. [CrossRef] [PubMed]

14. Mishra, R.; Varshney, R.; Das, N.; Sircar, D.; Roy, P. Synthesis and characterization of gelatin-PVP polymer composite scaffold for potential application in bone tissue engineering. Eur. Polym. J. 2019, 119, 155-168. [CrossRef]

15. Kirdponpattara, S.; Phisalaphong, M.; Kongruang, S. Gelatin-bacterial cellulose composite sponges thermally cross-linked with glucose for tissue engineering applications. Carbohydr. Polym. 2017, 177, 361-368. [CrossRef]

16. Ye, S.; Jiang, L.; Su, C.; Zhu, Z.; Wen, Y.; Shao, W. Development of gelatin/bacterial cellulose composite sponges as potential natural wound dressings. Int. J. Biol. Macromol. 2019, 133, 148-155. [CrossRef]

17. Yang, X.; Yang, D.; Zhu, X.; Nie, J.; Ma, G. Electrospun and photocrosslinked gelatin/dextran-maleic anhydride composite fibers for tissue engineering. Eur. Polym. J. 2019, 113, 142-147. [CrossRef] 
18. Liu, P.; Shen, H.; Zhi, Y.; Si, J.; Shi, J.; Guo, L.; Shen, S.G. 3D bioprinting and in vitro study of bilayered membranous construct with human cells-laden alginate/gelatin composite hydrogels. Colloids Surf. B Biointerfaces 2019, 181, 1026-1034. [CrossRef]

19. Zhou, L.; Xu, T.; Yan, J.; Li, X.; Xie, Y.; Chen, H. Fabrication and characterization of matrine-loaded konjac glucomannan/fish gelatin composite hydrogel as antimicrobial wound dressing. Food Hydrocoll. 2020, 104, 105702. [CrossRef]

20. Liu, W.-C.; Wang, H.-Y.; Lee, T.-H.; Chung, R.-J. Gamma-poly glutamate/gelatin composite hydrogels crosslinked by proanthocyanidins for wound healing. Mater. Sci. Eng. C 2019, 101, 630-639. [CrossRef]

21. Li, D.; Ye, Y.; Li, D.; Li, X.; Mu, C. Biological properties of dialdehyde carboxymethyl cellulose crosslinked gelatin-PEG composite hydrogel fibers for wound dressings. Carbohydr. Polym. 2016, 137, 508-514. [CrossRef]

22. Alavarse, A.C.; de Oliveira Silva, F.W.; Colque, J.T.; da Silva, V.M.; Prieto, T.; Venancio, E.C.; Bonvent, J.-J. Tetracycline hydrochloride-loaded electrospun nanofibers mats based on PVA and chitosan for wound dressing. Mater. Sci. Eng. C 2017, 77, 271-281. [CrossRef]

23. Karuppuswamy, P.; Reddy Venugopal, J.; Navaneethan, B.; Luwang Laiva, A.; Ramakrishna, S. Polycaprolactone nanofibers for the controlled release of tetracycline hydrochloride. Mater. Lett. 2015, 141, 180-186. [CrossRef]

24. Yang, M.; Li, L.; Yu, S.; Liu, J.; Shi, J. High performance of alginate/polyvinyl alcohol composite film based on natural original melanin nanoparticles used as food thermal insulating and UV-vis block. Carbohydr. Polym. 2020, 233, 115884. [CrossRef]

25. da Silva Fernandes, R.; de Moura, M.R.; Glenn, G.M.; Aouada, F.A. Thermal, microstructural, and spectroscopic analysis of Ca2+ alginate/clay nanocomposite hydrogel beads. J. Mol. Liq. 2018, 265, 327-336. [CrossRef]

26. Németh, B.; Németh, Á.S.; Ujhidy, A.; Tóth, J.; Trif, L.; Gyenis, J.; Feczkó, T. Fully bio-originated latent heat storing calcium alginate microcapsules with high coconut oil loading. Sol. Energy 2018, 170, 314-322. [CrossRef]

27. Correia, D.M.; Padrão, J.; Rodrigues, L.R.; Dourado, F.; Lanceros-Méndez, S.; Sencadas, V. Thermal and hydrolytic degradation of electrospun fish gelatin membranes. Polym. Test. 2013, 32, 995-1000. [CrossRef]

28. Deng, L.; Li, Y.; Zhang, A.; Zhang, H. Characterization and physical properties of electrospun gelatin nanofibrous films by incorporation of nano-hydroxyapatite. Food Hydrocoll. 2020, 103, 105640. [CrossRef]

29. Xiao, Q.; Gu, X.; Tan, S. Drying process of sodium alginate films studied by two-dimensional correlation ATR-FTIR spectroscopy. Food Chem. 2014, 164, 179-184. [CrossRef]

30. Hua, S.; Ma, H.; Li, X.; Yang, H.; Wang, A. pH-sensitive sodium alginate/poly(vinyl alcohol) hydrogel beads prepared by combined $\mathrm{Ca}^{2+}$ crosslinking and freeze-thawing cycles for controlled release of diclofenac sodium. Int. J. Biol. Macromol. 2010, 46, 517-523. [CrossRef]

31. Shao, W.; Wang, S.; Liu, X.; Liu, H.; Wu, J.; Zhang, R.; Min, H.; Huang, M. Tetracycline hydrochloride loaded regenerated cellulose composite membranes with controlled release and efficient antibacterial performance. RSC Adv. 2016, 6, 3068-3073. [CrossRef]

(C) 2020 by the authors. Licensee MDPI, Basel, Switzerland. This article is an open access article distributed under the terms and conditions of the Creative Commons Attribution (CC BY) license (http://creativecommons.org/licenses/by/4.0/). 